\title{
El concepto general de enfermedad. Revisión, crítica y propuesta Segunda parte: Carencias y defectos en los intentos por lograr una definición general de enfermedad
}

\author{
ADOLFO PEÑA, OFELIA PACO \\ Departamento Médico de la Asociación Cristiana de Jóvenes, YMCA.
}

\begin{abstract}
RESUMEN
Esta segunda entrega está dedicada a señalar y esclarecer algunas omisiones que, a nuestra opinión, exhiben las diversas propuestas de definición de enfermedad. Nuestra crítica espera revirar el interés hacia el uso de diversos conceptos, teorías y metateorías que bien pueden contribuir a la mejor ejecución de un modelo teórico de la enfermedad. El análisis de los conceptos, el uso de la teoría general de sistemas, de la modelización matemática y del materialismo emergentista, mostrarán que alcanzar un concepto general de enfermedad no es posible sólo acudiendo a la parcela de la investigación empírica (científica).
\end{abstract}

Palabras claves: Enfermedad; modelos teórico; teoría de sistemas.

\section{LACKS AND DEFECTS IN THE INTENTS TO ACHIEVE A GENERAL DEFINITION OF DISEASE SUMMARY}

This second delivery is dedicated to point out and clarify some omissions that to our opinion the diverse proposals of disease concept exhibit. This critic hopes to orientate the interest toward the use of diverse concepts, theories, and metatheories that may contribute to the best execution of a theoretical model of disease. The analysis of the concepts, the utilization of the general theory of systems, mathematical models, and emergentism theory will demonstrate that to reach a general concept of disease is not possible only by going to the parcel of empiric investigation.

Key words: Disease; models, theoretical; systems theory.

\section{Introducción}

Casi siempre en nuestras escuelas de medicina se nos enseña que la enfermedad no posee existencia real, sino tan sólo abstracta; "no existen enfermedades sino enfermos", suele escucharse. En algunos textos de lengua inglesa $\left({ }^{1}\right)$ se remarca esta diferencia, al asignar significado dispar a las palabras disease e illness. La

Correspondencia:

Dr. Adolfo Peña Salazar

Choquehuanca 208

Lima 32, Perú

E-mail: adolfoinquiry@yahoo.com primera, se dice, designa el conjunto de abstracciones, sin existencia física; por ejemplo, la tuberculosis descrita en los textos de patología. La segunda illness, caracteriza el padecimiento, la experiencia personal de aflicción, es decir, el proceso real.

Esa distinción ontológica-semántica continúa motivando nuestra forma de entender y "aceptar" la enfermedad. En la revisión que precedió $\left({ }^{2}\right)$, hemos visto que la discusión de cómo definir enfermedad continúa alrededor de la dicotomía naturalista-valorativa. Empero, pocos de los artículos publicados en prestigiosas revistas internacionales abordan el problema previo de 
deslindar y caracterizar la naturaleza ontológica de los objetos conceptuales. Denunciando tal omisión, iniciamos esta segunda entrega, dedicada a señalar y esclarecer algunos "errores" que, a nuestra opinión, han sido perpetrados en los diversos artículos especializados...

\section{La falta de un correcto entendimiento de los objetos conceptuales}

¿Qué es un concepto? ¿Es un ente propio? ¿Sólo un signo o una descripción arbitraria? Aquella discusión ha sido central a través de los tiempos, su influencia sobre las ciencias no ha sido marginal ni mucho menos inocua. Tanto de forma directa como indirecta las ideas acerca de la naturaleza de los objetos conceptuales han contribuido en el acierto o en el fracaso. En el caso de la definición general de enfermedad, pareciese que su contribución no ha sido muy "reflexiva" sino implícita, hasta casi inconsciente; aquí intentaremos revelarnos frente a dicho silencio.

\section{¿Cuál es la naturaleza de los conceptos?}

Desde Platón, los más grandes pensadores han afrontado esta interrogante. Así, para el platonismo, los objetos conceptuales son seres ideales que existen de por sí, independientemente del mundo físico; más tarde Descartes todavía otorgaba aquella jerarquía, gracias a su famoso "cogito, ergo sum" (pienso, luego existo).

Una respuesta diferente estuvo a cargo de los empiristas ingleses Locke y Hume $\left(^{3}\right)$. Para ellos, los objetos conceptuales son objetos mentales y existen al igual que las demás ideas, o sea, como sensaciones o imágenes; la experiencia sensorial los impone y los crea.

Kant plantea que los pensamientos pueden ocurrir sólo como producto de la interacción entre la mente y la realidad externa de la experiencia. Hegel fue un paso más allá y reconoció que el mundo externo puede influenciar las categorías y demás rótulos que usamos, pero no puede crearlas sobre nuestra mente cual tabla rasa (Hume), ni tampoco ser su consecuencia (Descartes).

Ludwing Wittgenstein, el influyente filósofo del Círculo de Viena, propone que los conceptos no son sólo ideas fijas o absolutas, sino definibles a través de reglas lógicas y referenciales. Un concepto -ya sea abstracto, como la verdad o mundano como la enfermedad- emerge como materia de "semejanza familiar"; por ejemplo, la malaria "sería" más parecido a enfermedad que el alcoholismo $\left({ }^{3}\right)$. Wittgenstein propone que los conceptos son derivados de aquellas semejanzas familiares, no de un conjunto de atributos fijos.

Actualmente, según Mario Bunge (pp. 50-61) $\left({ }^{4}\right)$, los objetos conceptuales no deben entenderse ni como entes, como lo cree el platonismo, ni tampoco como procesos cerebrales (pensamientos, percepciones, experiencias), como lo cree el empirismo; sino como objetos que poseen una naturaleza única e irreductible [existencia conceptual]. Su presencia es de alguna forma convencional o fingida, dado que concurren bajo un determinado contexto. Empero, si se refieren a hechos concretos y reales, como en las ciencias fácticas, no podrán ser arbitrarios. Debemos de aceptar entonces, dos tipos de existencia: la real (de carne y hueso) y la conceptual (creada a partir de una mente inteligente). Los objetos de la primera simplemente existen, y puesto que lo hacen independientemente de los humanos, son susceptibles de poseer propiedades físicas, estados y formas. El humano, gracias a sus sentidos, podrá percibirlas, para luego intentar conocerlas, explicarlas y representarlas, pero, al hacerlo, no podrá ni deberá asignarles propiedades que no le correspondan. Ambas clases de objetos poseen propiedades no intercambiables, por ejemplo, los conceptuales, a pesar que representen a los concretos, no podrán sentir, envejecer, enfermar o cambiar de estado; así como los objetos concretos, no podrán ser ni malos ni buenos, ni útiles ni inútiles, ni verdaderos ni falsos; por ello podemos conocerlos tal y como son, sin ataduras subjetivas o relativistas. 


\section{¿Cómo se estructuran los objetos conceptuales en las ciencias?}

En las ciencias, los objetos conceptuales (llamados más propiamente constructos) pueden ser agrupados en conceptos, que constituyen las unidades irreductibles. Proposiciones, los constructos que satisfacen algún cálculo proposicional, y además consiguen ser evaluados en lo que respecta a su grado de verdad, las hipótesis y los enunciados, son ejemplos. Y las teorías, sistemas cerrados e interdependientes de proposiciones, las cuales pretenden dar explicación a un segmento de la realidad y utilizan el método científico en ese intento (pp. 51-52) $\left({ }^{4}\right)$.

El conocimiento de la estructura teórica de las ciencias nos ayuda a dilucidar lo siguiente:

- Las proposiciones deben distinguirse en enunciados universales, particulares y singulares (pp. 60-62) $\left(^{5}\right)$. Un enunciado universal es el que se refiere a todos los elementos de una clase determinada. Uno particular, el que lo hace a algunos de los elementos de ella. Singular el que hace mención de un elemento dado, un individuo. Las leyes naturales se las considera enunciados estrictamente universales, por ejemplo.

Esta distinción se encuentra en estrecha conexión con la existencia de conceptos universales e individuales. Por ejemplo, la enfermedad linfoma de Hodking o el síndrome de DiGeorge, son conceptos o nombres universales; el paciente Juan Pérez o Ricardo DiGeorge, son conceptos individuales.

Aunque aparentemente sencilla, la aclaración anterior conlleva a implicancias importantes. Todas las aplicaciones de la ciencia se apoyan en inferencias que, partiendo de hipótesis científicas (universales), llegan a casos singulares. La práctica médica no puede escapar a ello. Por ejemplo, si el paciente Ricardo DiGeorge acude al hospital por presentar tumoraciones axilares, fiebre y prurito, es justificable de ser diagnosticado de linfoma, ya que se confía en las generalizaciones semiológicas propuestas y harto contrastadas.
- Por otra parte, los modelos teóricos (conjunto de proposiciones interdependientes que representan "operacionalmente" un aspecto de la realidad y que se encuentran engarzadas en las teorías científicas) constituyen el sinnúmero de modelos utilizados en medicina. Por ejemplo, el modelo de mosaico fluido $\left({ }^{6}\right)$, según el cual todas las células humanas están delimitadas por una bicapa lipídica y proteica, con propiedades comunes $y$ generales, como la fluidez y el libre movimiento lateral de sus proteínas. El modelo de regulación genética en procariotas, llamado operón $\left({ }^{7}\right)$, el modelo de doble hélice, de ácido base, hormona, neurotrasmisor, etc. Entonces ¿por qué no justificar una definición general de enfermedad?

- Las proposiciones deben ser diferenciadas en observacionales y no observacionales (pp. 5774) $\left(^{5}\right)$ (pp. 210) $\left(^{8}\right)$. Las primeras otorgan información sobre el momento y lugar en el que se realiza una observación. Las proposiciones no observacionales son aquellas cuyos términos son exclusivamente constructos hipotéticos, las hipótesis acerca de las cuatro fuerzas subatómicas, por ejemplo.

- Las teorías científicas son sistemas axiomáticos de proposiciones [hipótesis y enunciados] que se caracterizan por su ordenamiento lógico, de tal forma que a partir de la postulación de verdad de un número mínimo de hipótesis, se deduce la verdad de las demás (pp. 53-56) ${ }^{8}$ ). Los enunciados cuya verdad se postula se les llama axiomas y aquellos cuya verdad se deduce a través de una cadena de implicancias se les denomina teoremas. Parte de la contrastación de una teoría es lógica [asegurada por sus propiedades de coherencia o unicidad conceptual, simplicidad y consistencia externa (pp.146-150) $\left.\left({ }^{9}\right)\right]$. Otra es empírica, al ser necesario establecer que las proposiciones deducidas como teoremas corresponden al comportamiento efectivo de los hechos. Así, si corresponden, se dice que la teoría es, además de lógicamente correcta, empíricamente verdadera [gracias a sus propiedades de interpretabilidad empírica, representatividad, capacidad predictiva, escrutabilidad y especialmente refutabilidad (pp. 150-161) $\left.\left(^{9}\right)\right]$. 
Las teorías se ponen a prueba gracias a sus hipótesis de menor grado de generalidad (observacionales), y éstas a través del uso de los conceptos individuales. Así, si estas observaciones no se prueban, la teoría -sus proposiciones no observacionales- no queda contrastada y en última instancia no puede afirmarse su valor de verdad.

¿Por qué estudiar todo aquello? Porque las ciencias no son cúmulos de datos, sino básicamente sistemas teóricos, siendo su contenido los objetos conceptuales y su referente los objetos materiales; esta distinción es vital a fin de no crear confusiones iniciales, causantes de tesis poco válidas. Para el caso que nos interesa, al conocer mejor estos temas, podríamos obtener las siguientes recompensas:

- PRIMERO.- Cualquier definición de enfermedad será un constructo, dado que es producto de la mente (cerebro) de algún humano o de muchos.

- SEGUNDO.- Tal constructo no puede ser solamente un concepto, ya que si pretende ser la explicación de un aspecto de la realidad (como lo es el estado patológico), debe nutrirse de proposiciones que pongan a prueba su grado de verdad.

- TERCERO.- No solamente será una proposición, puesto que la complejidad del enfermar no puede ser explicada a través de una sola proposición o hipótesis observacional, sino de muchas.

- CUARTO.- Una definición general de enfermedad debe nutrirse de muchas proposiciones tanto observacionales (las de menor grado de generalidad) como no observacionales (las de mayor grado de generalidad.) La interdependencia sistémica de ambas garantizará la coherencia y exactitud del constructo, que en última instancia se convertirá en un modelo teórico, que además deberá ser científico si no quiere perderse los miles de datos de información que ya existen.

- QUINTO.- Debemos aspirar a elaborar una definición general de enfermedad que sea: a. Una representación conceptual, simbólica y aproximada del estado de un sistema biológico complejo.

b. Un modelo teórico que refiera sólo unos cuantos "aspectos clave" comunes y generales a todos los estados enfermos, y no sólo refiera los detalles individuales del sistema.

c. Que sea convencional pero no arbitrario.

d. Que no sea ambiguo y solamente literario, que recurra a términos teóricos definidos (como los de estado y propiedad) y que recurra a la modelización matemática y lógica para ser más exacto.

e. Que no pretenda ser una fotografía instantánea, ni una representación gráfica o pictórica del enfermar, puesto que estará "construida" de proposiciones y no de gráficos, ni de esquemas.

f. Que sea entendible y coherente con las leyes biológicas generales harto conocidas y contrastadas.

- SEXTO.- La mayoría de autores, como De Vitto $\left({ }^{10}\right)$, Rudnick $\left({ }^{11}\right)$, Fulford $\left({ }^{12}\right)$, Calguilhem $\left({ }^{13}\right)$, Scadding $\left({ }^{14}\right)$, Lenoox $\left({ }^{15}\right)$, y Temple $\left({ }^{16}\right)$, comenten un error ontológico al atribuir una propiedad no física como el valor, a estados físicos y materiales, como son los estados de salud y enfermedad. Al referirse sobre la imposibilidad de determinar qué es enfermedad sin usar criterios valorativos-normativos, y al referirse (cito textualmente) "In essence, the concepts of health and disease have both evaluative and value-neutral components that are individually necessary but only jointly sufficient for determining just what is illness" (pp. 540) $\left({ }^{10}\right)$. [En esencia, los conceptos de salud y enfermedad, ambos poseen componentes valorativos y valor-neutral que son individualmente necesarios, pero que sólo unidos determinan suficientemente qué es enfermedad], estos "especialistas" elaboran objetos ontológicamente malformados, invalidando así sus propuestas de definición.

Por poco creíble que parezca, la literatura en prestigiosas revistas está plagada de errores 
como ese. Lennox $\left({ }^{15}\right)$, por ejemplo, como revisáramos en el artículo anterior $\left({ }^{2}\right)$, no sólo propone que la salud y la enfermedad sean conceptos cargados de valor, sino que va más lejos; propone que tales "valores" son "objetivos" (es decir, reales, no conceptuales.)

Médicos peruanos tampoco han podido escapar a ese error; por ejemplo, el Dr. Gonzáles Diego $\left({ }^{17}\right)$ comenta nada menos que en un simposio de filosofía de la medicina: "Me inclino a pensar que la salud es una dimensión, la salud individual es la belleza de la existencia y la experiencia. Un equilibrio entre el ambiente, la conciencia, el espíritu, nuestras endorfinas, nuestra imagen propia: Por eso tengo la convicción que la salud individual es una metáfo$r a$ " [ino puede haber mejor ejemplo de desliz ontológico!]

- SÉPTIMO.- No es admisible la posición de Hesslow $\left({ }^{18}\right)$, quien afirma la imposibilidad de un concepto general de enfermedad.

- OCTAVO.- Las diversas tesis publicadas no llegan a constituir ni teorías, ni modelos teóricos, a lo más son opiniones, quizás doctrinas o "marcos" teóricos. Aunque constituidas por muchas proposiciones y oraciones con un referente común: la enfermedad. Sus proposiciones no han sido claramente especificadas ni organizadas en un sistema hipotético-deductivo interdependiente; sus interpretaciones no son ni semánticas ni epistemológicas, sino sólo literarias.

\section{La falta de una caracterización precisa de la "noción" estado}

Otro gran defecto en los ensayos dedicados a definir enfermedad, es su ambigüedad. Así, ninguno de ellos, desde Bernard en 1860 hasta Temple en el 2001, ha dedicado una caracterización precisa del concepto estado; en el mejor de los casos se remiten a utilizar aquella palabra, pero no la definen con rigor. Por ejemplo, Boorse $\left({ }^{19}\right)$, quien ha escrito todo un libro acerca del tema, no establece qué son esos "estados" al que hace referencia: "La enfermedad es un tipo de estado interno en el cual existe un impedimento del funcionamiento normal, es decir, una reducción de una o más habilidades funcionales por debajo de lo típicamente eficiente o en limitación sobre la habilidad funcional causado por agentes ambientales".

Mientras que ya muchas teorías científicas utilizan la matemática de conjuntos para explicar y entender qué es "estado", en medicina (al menos en lo que respecta a definir enfermedad) se hace caso omiso del uso de las VARIABLES DE ESTADO, de las representaciones de los ESPACIOS DE ESTADO y de las FUNCIONES MATEMÁTICAS. El problema, pues, de muchas de las definiciones propuestas es que, aunque pueden ser consideradas como "correctas" sólo lo serán entre comillas, debido a que dependen de conceptos no elucidados previamente.

\section{La falta de utilización de la teoría general de sistemas en la definición de enfermedad}

La teoría general de sistemas, desde que se fundó con Bertalanffy hace cincuenta años $\left({ }^{20-}\right.$ ${ }^{21}$ ), ha recibido gran reconocimiento en diferentes disciplinas, tanto científicas como tecnológicas. Concebida como una teoría de alto grado de generalidad, de corte lógico-matemático, propone que la realidad se estudie como un sistema de conexiones cualitativamente determinado, en donde cada elemento se analice como parte de un todo y a la vez como un subsistema en sí mismo $\left({ }^{20-21}\right)$.

Gracias a su planteamiento, los frutos posteriores a su uso han sido harto producidos, con publicaciones en revistas científicas que cubren áreas desde física, química y biología, hasta sociología, economía y educación. Actualmente, la Society for General Systems Research (Sociedad para la investigación general de sistemas) es una de las sociedades científicas más relevantes $\left({ }^{22}\right)$.

A pesar de su enorme difusión y aunque paradójicamente su fundador, Bertalanffy, fue un biólogo "contiguo" a la medicina, la teoría general 
de sistemas parece ser completamente ignorada. En ningún artículo publicado en los últimos veinte años se encuentra siquiera un titulo dedicado a intentar hacer uso de dicha teoría a fin de elaborar una definición de enfermedad. Así pues, conceptos como sistema, elementos de un sistema, estructura de un sistema, ESTADOS, PROCESOS y PROPIEDADES, son inexplorados. He aquí una razón del por qué los filósofos de la medicina no pueden dar explicación del concepto estado y, en consecuencia, no pueden dar explicación de lo que es enfermedad.

\section{Las definiciones actuales de enfermedad no hacen uso del materialismo emergentista compatible con la estructura en niveles de la realidad}

La "hipótesis" de "emergencia" permite explicar el por qué no se puede elucidar ciertas propiedades de los sistemas complejos únicamente a través del completo y detallado estudio de sus partes. El materialismo emergentista se está convirtiendo en los últimos años en un enfoque influyente $\left({ }^{23-24}\right)$, sobretodo, en psicología y neurociencia, ya que entre otras cosas está logrando crear el "armazón intelectual" sobre el cual la investigación científica pueda dar cuenta de conceptos tan difíciles como los de mente, vida y organismo $\left({ }^{25}\right)$.

Los orígenes del concepto de emergencia pueden remontarse, según Sawyer $\left({ }^{23}\right)$, hasta el siglo pasado, cuando el filósofo George Henry Lewes (1877) en una crítica que hacía a la teoría de la causación de Hume, halló necesario distinguir entre dos tipos de efectos: resultantes y emergentes. "Aunque cada efecto es el resultante de sus componentes, el producto de sus factores no siempre puede trazar los pasos del proceso, así como en el producto el modo de operación de cada factor. En este último caso, yo propongo llamar al efecto un emergente. Ello emerge por la agencia combinada, pero en una forma que no muestra los agentes en acción" ${ }^{(23)}$. Posteriormente, en los años 20s, esta idea fue compartida por más filósofos, sobretodo por filósofos de la biología, entre ellos Lloyd Morgan, quien postuló la emergencia de los niveles organizacionales. Ya a mediados del siglo $\mathrm{XX}$, el concepto de emergencia toma un nuevo "empuje", integrándose parcialmente en la teoría general de sistemas, y mediante ella a diferentes disciplinas.

Actualmente, la hipótesis de emergencia consiste básicamente en sostener que todos los objetos y sistemas poseen propiedades (características) que pueden ser RESULTANTES (HEREDITARIAS) si son derivadas íntegramente a partir de sus partes (de forma sumatoria) o EMERGENTES (COLECTIVAS) si sólo lo posee el sistema, pero no las partes ${ }^{(23-25)}$.

Es de señalar que la emergencia se debe entender como hecho real y concreto y no sólo como un mero arreglo o estrategia cognitiva, ya que toda emergencia depende no sólo de ideas, sino de las cosas reales que la "contienen" (pp. 42-46) ${ }^{25}$ ). Es decir, el emergentismo racional afirma tanto la emergencia óntica, como su reductibilidad gnoseológica o explicabilidad.

A fin de mostrar la utilidad de dicho enfoque, veamos el ejemplo más conocido $\left({ }^{26}\right)$ : El agua, como sabemos, puede encontrarse macroscópicamente en diferentes estados físicos (sólido, líquido y gaseoso); empero, sus moléculas que la constituyen no poseen tales propiedades; así, una molécula aislada de $\mathrm{H}_{2} \mathrm{O}$ no es ni "fluida" ni "gaseosa" ni "gélida". Estos estados se explican por la interacción electrostática a través de los enlaces de hidrógeno -la interacción electromagnética entre átomos de hidrógeno y pares de electrones compartidos de otros átomos- entre las moléculas de agua, enlaces que pueden tomar diferente angulación, dependiendo de la temperatura del medio: agudo para el estado líquido y recto para el sólido $\left({ }^{27}\right)$. Es entonces a partir de la organización particular e interdependiente de los elementos (moléculas de $\mathrm{H}_{2} \mathrm{O}$ ) que se permite la "emergencia" de una propiedad perteneciente solamente al agua como sistema. Podemos concluir luego, que tal propiedad líquida o sólida, no se "encuentra" en la molécula $\mathrm{H}_{2} \mathrm{O}$ ni en los 
enlaces de hidrógeno, tampoco en los átomos, sino en el sistema que se crea al unirse miles o millones de moléculas. La fluidez del agua es entonces una propiedad emergente, se explica por sus componentes (átomos de $\mathrm{H}$ y de $\mathrm{O}$, moléculas de $\mathrm{H}_{2} \mathrm{O}$ y enlaces electromagnéticos), pero no es compartida por ninguno de ellos, sino que pertenece a un sistema de un nivel jerárquico superior: el agua.

La biología, y por ende la medicina, está plagada de ejemplos como el que acabamos de ver. Propiedades como la sexualidad, la mente, la respiración, visión, audición, digestión, estructura proteica, contracción muscular, y la conducta son emergentes. No pueden evitar serlo, a fin y al cabo, el "mundo" es sistémico, y no simple.

Finalmente postulamos que lo que nos interesa en este ensayo: LA ENFERMEDAD también es de carácter EMERGENTE.

\section{La ausencia de un concepto claro y sistémico de organismo}

No podemos definir enfermedad como un estado, sino esclarecemos qué es estado. Adicionalmente, este ultimo concepto es dependiente de su referente objetivo, ya que debe ser el "estado" de algo, de una cosa u objeto concreto (en nuestro caso, el ser humano.) Entonces, una definición al menos aproximada de organismo ha de imponerse. Lamentablemente aquí encontramos otra "ausencia" en las diversas publicaciones.

Aquí, algunos conceptos y postulados virtualmente "ignorados" $\left({ }^{20-21}\right)$ :

a) Sistema.- Objeto complejo compuesto de ítems interdependientes.

b) Elementos.- Los componentes de cada sistema.

c) Propiedades.- Los atributos o características de cada objeto, subsistema o sistema. Pueden ser resultantes, si son compartidas por todos los elementos, o emergentes si son "únicos". d) Estructura.- Las relaciones que ligan a los elementos de un sistema como un todo; puede ser simple o compleja, dependiendo del número y tipo de interrelaciones entre las partes. Los sistemas complejos tendrán diferentes niveles jerárquicos en su estructura.

e) Estados y procesos.- El estado de un sistema queda definido por las propiedades que sus elementos muestran en un determinado tiempo. El valor de sus variables de estado lo caracterizan. Los cambios de un estado a otro, son los procesos o el proceso del sistema.

f) Ambiente.- Es el medio (porción de la realidad o del universo) con el cual un determinado sistema interactúa, ya sea introduciendo (input) o eliminando (output) información, objetos o cualquier otra cosa.

Cinco postulados adicionales $\left({ }^{28}\right)$ :

i) Todas las cosas, sean concretas o abstractas, son sistemas o componentes actuales, o potenciales de un sistema.

ii) Los sistemas poseen características sistémicas (emergentes) que son ausentes en sus componentes.

iii) Todos los problemas deben ser abordados de forma sistémica, más que de manera sectorial.

iv) Todas las ideas deben ser agrupadas y organizadas en sistemas (teorías).

v) Las pruebas de cualquier cosa, ya sea idea o artefacto, asume la validez de los otros postulados, los cuales son tomados como lineamientos.

Tomando estos conceptos y postulados, se puede definir organismo viviente como aquel quimiosistema semiabierto y autocontrolado que toma del medio ambiente la materia y la energía que intervienen en sus reacciones, que sintetiza todos sus demás componentes y que se reproduce, muta y evoluciona. Difieren de lo inanimado, aunque se enraízan en éste y emergen de él en un proceso histórico. Son vivientes, porque poseen propiedades biológicas (repro- 
ducción, mutación, evolución, etc.) que sólo les pertenece a ellos como biosistemas...

Y como todo biosistema, algunos de sus estados serán saludables y otros serán ENFERMOS....

\section{La falta de la modelización matemática}

Después de obtener decenas de artículos a través de Medline, utilizando las palabras clave de búsqueda: disease definition, illness, theory of disease, y health; sólo encontramos a un autor, Sadegh-Zadeh $\left(^{29}\right)$, que utilice la modelización matemática para esclarecer su modelo de enfermedad. Esto muestra que en medicina -a diferencia de otras tecnologías- los investigadores biomédicos sólo reservan la matemática para la investigación empírica (ya sea básica o clínica), pero no acuden a ella para "esclarecer" sus ideas y conceptos centrales.

Utilizar la matemática no responde a un mero juego mental, responde esencialmente, a generalizar y operacionalizar mejor conceptos y proposiciones no individuales, posibilitando crear un esqueleto formal el cual pueda "rellenarse" con cualquier contenido empírico (pp. 170,171) $\left({ }^{4}\right)$. La matematización del concepto general de enfermedad incrementaría la exactitud y, por lo tanto, la claridad de las ideas. En contraste, los actuales conceptos literarios muy ricos en inferencias laboriosas y a menudo inseguras, no pueden especificar bien sus premisas fundamentales.

Un modelo matemático se puede ordenar mejor, y en particular, se puede axiomatizar, otorgando precisión y poder deductivo. Tal ordenamiento lógico facilita su contrastación empírica y aumenta su verificabilidad, sin la necesidad de acudir a la ideología o a la doctrina (pp. 171) $\left({ }^{4}\right)$.

Parece que algunas de las respuestas no provienen necesariamente de los investigadores biomédicos. Es un filósofo, Mario Bunge (pp. 239-241) $\left({ }^{4}\right)$, quien ha adelantado en "llenar" algunos de los vacíos de las publicaciones biomédicas mundiales, el uso de la modelización matemática y del enfoque sistémico para definir enfermedad ha sido señalado por él $\left({ }^{30}\right)$.

\section{Conclusión}

A pesar del desarrollo científico, el concepto de enfermedad todavía conlleva dificultades, quizá la falta de interés o de iniciativa hacia el estudio de otras disciplinas ha contribuido a esta limitación. Se hace necesario integrar las metateorías más productivas con el análisis filosófico más crítico. Dado su alto nivel de abstracción, la elaboración de un modelo teórico de enfermedad no plantea tarea sencilla, pero esta tarea será aun más difícil si no perdemos el miedo a la introducción de hipótesis que contengan factores no observables, y si no luchamos por la organización lógica de los postulados. De no conseguir una estructura lógica axiomática será más difícil reconocer los componentes esenciales que nos permitan la mejor crítica y revisión.

\section{BIBLIOGRAFÍA}

1. Delp MH, Manning RT. Major's Physical Diagnosis. An Introduction to the Clinical Process, ninth edition. Philadelphia: W.B. Saunders Co. 1981: 1-11, 42-3.

2. Peña A, Paco O. El concepto general de enfermedad. Revisión, crítica y propuesta. Primera parte. Anales de la Facultad de Medicina 2002; 63: 223-32.

3. Norman GR. The epistemology of clinical reasoning. Perspectives from philosophy, psychology, and neuroscience. Acad Med 2000; 75:(Supl.) S127-S135.

4. Bunge MA. Epistemología. Curso de actualización. Barcelona: Ariel; 1985.

5. Popper KR, Sánchez de Zavala (trad.) La Lógica de la Investigación Científica. Madrid: Tecnos; 1962. Originalmente publicado, en inglés: The Logic of Scientific Discovery, London: Hutchinson; 1959.

6. Singer SJ, Nicolson GL. The fluid mosaic model of the structure of cell membranes. Science 1972; 175: 720-30.

7. Jacob F, Monod J. Genetic regulatory mechanisms in the synthesis of proteins. J Mol Biol 1961; 3: 318-56.

8. Piscoya LH. Investigación Científica y Educacional. Un enfoque epistemológico 2da. ed. Lima: Amaru Editores; 1995.

9. Bunge MA, García JL y Sempere J (trads.) Teoria y Realidad. Barcelona: Ariel; 1981. 
10. De Vitto S. On the value-neutrality of the concepts of health and disease: unto the breach again. J Med Philos 2000; 25: 539-67.

11. Rudnick A. The ends of medical intervention and the demarcation of the normal from the pathological. J Med Philos 2000; 25: 569-80.

12. Fulford K. What is (mental) disease?: An open letter to Christopher Boorse. Journal of Medical Ethics 2001; 27: 80-6.

13. Canguilhem G. Lo Normal y lo Patológico, Buenos Aires: Siglo XXI; 1971. Originalmente publicado, en francés: Le normal et le pathologique. Paris: Presses Universitaries de France, 1956.

14. Scadding JG. Essentialism and nominalism in medicine: logic of diagnosis in disease terminology. Lancet 1996; 348: 594-600.

15. Lennox JG. Health as an objetive value. J Med Philos 1995; 20: 499-511.

16. Temple $\mathbf{L}$. Defining disease in the genomic era. Science 2001; 293: 807-8.

17. Gonzales D. La salud es una metáfora. En: Simposio Filosofía de la Medicina, Lima: fondo editorial de la UPCH; 2000: 19-28.

18. Hesslow G. Do we need a concept of disease? Theor Med 1993; 14: 1-14.

19. Boorse C. En: Humber J, Almeder R.(eds.) What is Disease? New Jersey: Humana Press; 1997.
20. Van Giegch J. Applied General systems Theory, 2da. Ed. New York: Harper and Row; 1978.

21. Voltes Bou. La Teoría General de Sistemas. Madrid: Editorial Hispano-Europea; 1978.

22. Society for General Systems Research. Disponible en: http://www.isss.org . Ingresado en diciembre del 2001.

23. Sawyer R. The emergence of creativity. Philos Psycho 1999; 12: 447-70.

24. Stadler M. Gestalt theory and synergetics: from psychophysical isomorphism to holistic emergentism. Philos Psycho 1994; 7: 211-227.

25. Bunge M. El Problema Mente-Cerebro. Un enfoque psicobiológico. 2da. Ed. Madrid: Tecnos; 1988. Originalmente publicado en inglés: The Mind-Body Problem. A psychobiological approach. Oxford, Pergamon Press; 1980.

26. Searle JR. Dos biológos y un físico en busca del alma. Mundo científico 1996, 170: 654-69.

27. Davison V. Biochemistry, $3^{\mathrm{a}}$. Ed. Philadelphia: Harwal Publishing; 1994.

28. Bunge M. Systemism: the alternative to individualism and holism. J Socio-Economics 2000; 29: 147-58.

29. Sadegh-Zadeh K. Fuzzy health, illness, and disease. J Med Philos 2000; 25: 605-38.

30. Peña A, Paco O. Un modelo teórico de enfermedad. Aceptado para publicación por la revista Anales de la Facultad de Medicina (en prensa). 